\title{
Fluid-structure interaction simulation of pulse propagation in arteries: numerical pitfalls and hemodynamic impact of a local stiffening
}

\begin{abstract}
When simulating the propagation of a pressure pulse in arteries, the discretization parameters (i.e. the time step size $\Delta t$ and the grid size $\Delta x$ ) need to be chosen carefully in order to avoid a decrease in amplitude of the traveling wave due to numerical dissipation. In this paper the effect of numerical dissipation is examined using a numerical fluid-structure interaction (FSI) model of the pulse propagation in an artery. More insight in the influence of the temporal and spatial resolution of the wave on the results of these simulations is gained using an analytical study in which the scalar linear onedimensional transport equation is considered. Although this model does not take into account the full complexity of the problem under consideration, the results can be used as a guidance for the selection of the numerical parameters. Furthermore, this analysis illustrates the difference in accuracy that can be obtained using a second-order implicit time integration scheme instead of a first-order scheme.

The results from the analytical and numerical studies are subsequently used to determine the settings necessary to obtain a grid and time step converged simulation of the wave propagation and reflection in a simplified model of an aorta with repaired aortic coarctation. This FSI model allows to study the hemodynamic impact of a stiff segment and demonstrates that the presence of a stiff segment has an important impact on a short pressure pulse, but has almost no influence on a physiological pressure pulse. This phenomenon is explained by analyzing the reflections induced by the stiff segment.
\end{abstract}

Keywords: Fluid-structure interaction, Numerical dissipation, Wave propagation, Pulse wave analysis, Aortic coarctation

\section{Introduction}

The measured blood pressure is the resultant of forward pressure waves, traveling from the heart towards the peripheral arteries and backward pressure waves, generated by reflections along the arterial path and in the periphery. The later reflected compression waves reach the heart, the less they generate an additional load on the heart. A compression wave reaching the heart in early systole (as is the case for older people with stiffened arteries or for patients with hypertension) leads to an increased systolic blood pressure and is a major risk factor for developing cardiovascular diseases $[1,2,3]$. The analysis of wave travel and reflection, sometimes referred to as 'pulse 
wave analysis', is increasingly used for the assessment of arterial and cardiovascular health and for the early diagnosis of cardiovascular risk.

One of the many possible applications of pulse wave analysis, is the study of wave reflections generated by a repaired aortic coarctation. Aortic coarctation is a congenital disease characterized by an obstructive narrowing of the upper descending aorta. The treatment can be minimally invasive using a stent and/or balloon catheter to dilate the coarctation zone [4, 5], or the narrow section can be removed surgically [6]. However, even after successful treatment a local stiffening remains, caused by the presence of the stent or the scar tissue. This stiffening generates an impedance mismatch and is a source of wave reflections that reach the heart fast, given the short distance to the heart [7]. Analysis of these wave reflections could thus be used to estimate the effect of the treatment of aortic coarctation on the load on the heart.

The study of wave reflections can either be based on experiments (using in vivo or in vitro pressure and velocity measurements), or on data obtained from computational models of various levels of complexity. With advances in computing power and numerical algorithms, computational simulations are extensively used for applications where experimental data are limited or unavailable. One-dimensional (1D) models are used to study wave propagation phenomena in the entire arterial tree or major parts of it (see, e.g. $[8,9,10,11])$ whereas local flow field details can only be obtained from threedimensional (3D) computational fluid dynamics or more complex fluid-structure interaction models (see, e.g. $[12,13,14])$. Provided that the model parameters faithfully represent the physical quantities of the arterial system and the numerical parameters are chosen carefully, these computational models can accurately predict the pressure and flow fields.

However, in many simulations, too little attention is paid to the proper selection of the numerical parameters. In that case considerable numerical dissipation and dispersion may influence the results and have a significant impact on the calculated waveforms and subsequent data interpretation. When simulating the propagation of a short pulse in an artery, for instance, the time step size needs to be carefully chosen in order to avoid a decrease in pulse amplitude that is not only caused by viscous dissipation $[15,16]$. Parameters that affect the numerical dissipation are the time step size $\Delta t$ and the grid size $\Delta x$, which are mostly chosen sufficiently large to reduce the calculation time, but should be adapted to the period of the present wave harmonics and their wavelength.

The aim of this study is threefold. First, a numerical study is performed to illustrate the effect of the previously mentioned parameters on the simulation results. Second, the FSI problem under consideration is simplified to the $1 \mathrm{D}$ blood flow in a flexible tube and a von Neumann analysis $[17,18]$ is presented in which the relation between the numerical dissipation and the discretization parameters is derived for a certain combination of discretization in space and time. The methodology followed in this study can, however, be used in a straightforward way to study other discretization schemes. Third, these results are applied to determine the numerical parameters necessary for the simulation of the pulse propagation in a locally stiffened tube (i.e. a simple model representative for an aorta with repaired aortic coarctation) and the hemodynamic impact of the stiff segment is studied. 


\section{Materials and methods}

\subsection{Numerical study}

To illustrate the effect of the discretization parameters, a numerical study is performed in which the propagation of a sinusoidal pressure pulse in a straight tube is simulated using a two-dimensional axisymmetric FSI model. Different time step sizes and grid sizes are used to analyze their influence on the numerical dissipation.

\subsubsection{FSI model}

The geometrical model consists of a two-dimensional axisymmetric tube with a length of $60 \mathrm{~cm}$, an inner diameter of $1.5 \mathrm{~cm}$ and a wall thickness of $0.15 \mathrm{~cm}$ (see Figure 1). At the inlet of the fluid domain a sinusoidal pressure pulse (period of $15 \mathrm{~ms}$, amplitude of $250 \mathrm{~Pa}$ ) is applied. At the outlet a reflection free boundary is implemented. This is done by imposing the following relation between the change in outlet pressure, $\Delta p(t)=p(t)-p(t-\Delta t)$, and outlet velocity $\Delta v(t)=v(t)-v(t-\Delta t)$ at two consecutive time steps.

$$
\Delta p=\rho_{f} c \Delta v
$$

In this equation $\rho_{f}$ represents the blood density and $c$ the wave speed. The latter one is adapted according to the pressure level by means of the Bramwell-Hill equation.

$$
c=\sqrt{\frac{d p}{\rho_{f} \frac{d A}{A}}}
$$

In this equation $A$ represents the cross section of the tube. $c$ is calculated in a separate structural analysis (Abaqus FEA) for different values of $p$ in a range from 500 to 1500 $\mathrm{Pa}$ and linear interpolation between these data points is performed.

Both ends of the tube wall are fixed in longitudinal direction. Blood is modeled as an incompressible and Newtonian fluid (density $1050 \mathrm{~kg} / \mathrm{m}^{3}$, viscosity $3 \mathrm{mPas}$ ) and the flow is laminar. A linear elastic material model is used to describe the wall behavior (Young's modulus $250 \mathrm{kPa}$, Poisson's ratio 0.49 , density $1200 \mathrm{~kg} / \mathrm{m}^{3}$ ). These parameters yield a pulse wave velocity of $4.86 \mathrm{~m} / \mathrm{s}$ and thus a wavelength of $0.073 \mathrm{~m}$ in unloaded state. All simulations are performed until time periodic solutions are obtained (i.e. until the change in amplitude of the pressure wave at the end of the tube at two consecutive periods becomes less than $0.2 \%$ ).

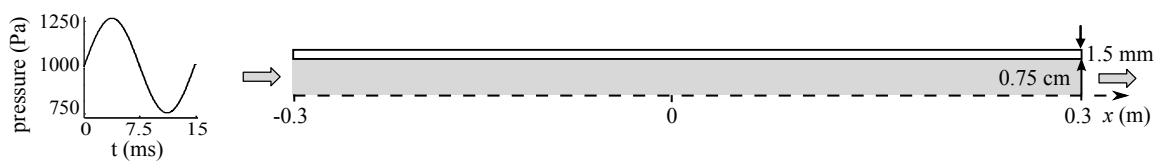

Figure 1: Two-dimensional axisymmetric FSI model.

The FSI problem is solved in a partitioned way, as such the equations for the blood flow and the deformation of the structure are solved separately. The flow equations 
are solved using a pressure-based solver (ANSYS Fluent), which computes the flow in a segregated manner using the SIMPLE algorithm $[19,20]$. A second-order upwind discretization scheme is used for the convective term in the momentum equation. The diffusion terms are central differenced and are second-order accurate. For the temporal discretization of the flow equations a second-order implicit scheme is applied. A finite element package (Abaqus FEA) is applied to calculate the wall deformations. The nonlinear equilibrium equations are solved using the Newton method with a fixed time increment. For the temporal discretization of the structure the operator defined by Hilber, Hughes and Taylor [21] is applied, which is an unconditionally stable scheme and possesses controllable algorithmic damping properties.

To obtain an accurate calculation of the stress on the fluid-structure interface, the flow equations are solved in an Arbitrary Lagrangian-Eulerian formulation on a deforming mesh. The structural equations are solved in a Lagrangian frame.

Besides a flow solver (ANSYS Fluent) and a structural solver (Abaqus FEA), a coupling algorithm is required to take into account the interaction between the flow and the motion of the structure. Due to the flexibility of the arterial wall and the comparable density of the blood and the structure, this interaction is strong. A stable and efficient algorithm is therefore needed to solve the coupled problem within an acceptable time. In this work a quasi-Newton algorithm with an approximation for the inverse of the Jacobian (IQN-ILS) is used, which requires fewer coupling iterations per time step than dynamic relaxation techniques [22].

\subsubsection{Governing equations}

The equations that describe the unsteady flow of the Newtonian, incompressible, viscous fluid are the conservation of mass and the Navier-Stokes equations, given by

$$
\begin{aligned}
\nabla \cdot \boldsymbol{v}_{f} & =0 \\
\rho_{f} \frac{\partial \boldsymbol{v}_{f}}{\partial t}+\rho_{f} \nabla .\left(\boldsymbol{v}_{f} \boldsymbol{v}_{f}\right) & =\rho_{f} \boldsymbol{f}_{f}+\nabla \cdot \sigma_{f}
\end{aligned}
$$

with $v_{f}$ the flow velocity, $t$ the time and $f_{f}$ the body forces per unit of mass on the fluid. The stress tensor $\sigma_{f}$ is defined as

$$
\sigma_{f}=-p \boldsymbol{I}+\boldsymbol{\tau}
$$

with $\boldsymbol{I}$ the unit tensor and $\tau$ the viscous stress tensor.

The velocity of the structure $\boldsymbol{v}_{s}$ is determined by the Cauchy momentum equation that describes the non-relativistic momentum transport in any continuum.

$$
\rho_{s} \frac{D \boldsymbol{v}_{s}}{D t}=\rho_{s} \boldsymbol{f}_{s}+\nabla \cdot \boldsymbol{\sigma}_{s}
$$

In this equation $\rho_{s}$ represents the structural density, $\sigma_{s}$ the Cauchy stress tensor and $f_{s}$ the body forces per unit of mass on the structure. The notation $D / D t$ refers to the material derivative.

The equilibrium conditions on the fluid-structure interface are the kinematic condition

$$
v_{f}=v_{s}
$$


and the dynamic condition

$$
\sigma_{f} \cdot \boldsymbol{n}_{f}=-\sigma_{s} \cdot n_{s} .
$$

$\boldsymbol{n}_{f}$ and $\boldsymbol{n}_{s}$ represent the unit normal vector that points outwards from the fluid respectively the structural domain.

\subsection{Analytical study}

One possibility to examine the effect of the discretization parameters at the start of a simulation, is to perform a numerical study as described in section 2.1. However, this can be very time consuming as FSI simulations are characterized by a high computational cost.

Another possibility to gain insight in the influence of the temporal and spatial discretization on the numerical dissipation is to perform an analytical study in which the 2D or 3D FSI problem is simplified to the one-dimensional one and the scalar linear transport equation is considered. Even though the von Neumann analysis is a well documented topic, this option is often overlooked or unknown to researchers performing numerical FSI simulations. A brief description of the analysis is given in the next section. A more distinguished analysis can be found in [17, 18]. Although the model does not take into account the full complexity of the problem under consideration, the results can be used as a guidance for the selection of the numerical parameters.

\subsubsection{Transport equation}

The scalar linear one-dimensional transport equation is given by

$$
\frac{\partial u}{\partial t}=-c \frac{\partial u}{\partial x}
$$

where $c$ is the speed of propagation of the scalar $u$, which is a function of time $(t)$ and space $(x)$. Assuming that $c$ is constant, the wave component of the solution with wavenumber $k$ can be written as

$$
u(x, t)=B e^{j k(x-c t)}
$$

with $B$ a constant and $j$ the imaginary unit. The wavenumber $k$ is inversely proportional to the wavelength $\lambda$, given by the product of the speed of propagation $c$ and the period of the wave $T$.

$$
k=\frac{2 \pi}{\lambda}=\frac{2 \pi}{c T}
$$

Eq. (10) can be split into a time dependent $\phi(t)$ and a space dependent part $\psi(x)$.

$$
u(x, t)=\phi(t) \psi(x)=\phi(t) e^{j k x}
$$

with

$$
\begin{cases}\phi(t) & =B e^{-j k c t} \\ \psi(x) & =e^{j k x}\end{cases}
$$




\subsubsection{Spatial discretization}

The artery through which the pressure pulse propagates is discretized in spatial intervals of equal length $\Delta x$. For the analysis, a second-order upwind discretization scheme [19] is used for the convective term in the transport equation, as this scheme will be used in the numerical simulations afterwards. Nevertheless, this analysis can be repeated for other schemes in a straightforward way. The spatial discretization of Eq. (9) is thus given by

$$
\frac{\partial u}{\partial t}=c\left[-\frac{3}{2} \frac{u(x)-u(x-\Delta x)}{\Delta x}+\frac{1}{2} \frac{u(x-\Delta x)-u(x-2 \Delta x)}{\Delta x}\right] .
$$

After substitution of Eq. (12), the spatially discretized transport equation becomes

$$
\begin{aligned}
\frac{\partial u}{\partial t} & =\frac{c}{\Delta x}\left[-\frac{3}{2}\left(e^{j k x}-e^{j k(x-\Delta x)}\right)+\frac{1}{2}\left(e^{j k(x-\Delta x)}-e^{j k(x-2 \Delta x)}\right)\right] \phi(t) \\
& =\frac{c}{\Delta x}\left[-\frac{3}{2}\left(1-e^{-j k \Delta x}\right)+\frac{1}{2}\left(e^{-j k \Delta x}-e^{-j k 2 \Delta x}\right)\right] \phi(t) e^{j k x} \\
& =\frac{c}{\Delta x} F \phi(t) e^{j k x}
\end{aligned}
$$

with

$$
F=-\frac{3}{2}\left(1-e^{-j \theta}\right)+\frac{1}{2}\left(e^{-j \theta}-e^{-2 j \theta}\right)
$$

and

$$
\theta=k \Delta x .
$$

\subsubsection{Time discretization}

Eq. (15) is subsequently discretized in time. Two schemes were applied, however, the analysis can easily be repeated for other discretization schemes. Using a secondorder implicit scheme, the time discretization of Eq. (15) is given by

$$
\frac{\frac{3}{2} u(t+\Delta t)-2 u(t)+\frac{1}{2} u(t-\Delta t)}{\Delta t}=\frac{c}{\Delta x} F \phi(t+\Delta t) e^{j k x} .
$$

This scheme is unconditionally stable and is well suited for the simulation of wave propagation.

To examine the effect of the accuracy of the time integration scheme, the numerical dissipation obtained with the second-order scheme is compared to the one obtained with a first-order scheme. Using the backward Euler scheme, the time discretization of Eq. (15) is given by

$$
\frac{u(t+\Delta t)-u(t)}{\Delta t}=\frac{c}{\Delta x} F \phi(t+\Delta t) e^{j k x} .
$$

Although this scheme is not very accurate, it is unconditionally stable and is implemented in most numerical solvers and is therefore used in many applications [15]. 
Substituting Eq. (12) and the Courant number, given by

$$
C=\frac{c \Delta t}{\Delta x}
$$

in the previous equations, Eqs. (18) and (19) respectively become

$$
\begin{gathered}
\frac{3}{2} \phi(t+\Delta t)-2 \phi(t)+\frac{1}{2} \phi(t-\Delta t)=C F \phi(t+\Delta t) \\
\phi(t+\Delta t)[1-C F]=\phi(t) .
\end{gathered}
$$

\subsubsection{Numerical dissipation}

Defining the amplification factor $G$ as

$$
G=\frac{\phi(t+\Delta t)}{\phi(t)}=\frac{\phi(t)}{\phi(t-\Delta t)}
$$

the numerical dissipation after one time step of a sine wave traveling with a constant speed can be calculated by

$$
1-|G|=1-\left|\frac{1}{2-\sqrt{1+2 C F}}\right|
$$

for the second-order scheme and by

$$
1-|G|=1-\left|\frac{1}{1-C F}\right|
$$

for the first-order scheme. The numerical dissipation after one time step is thus a function of two dimensionless parameters: $\Delta x / \lambda$, being a measure of the spatial resolution of the wave and $\Delta t / T$, which is a measure of the temporal resolution of the wave. Remark that, in some methods, due to restrictions in the Courant number, $\Delta x$ and $\Delta t$ cannot be chosen independently [23]. This is, however, not the case for the implicit method used in this analysis.

Usually, the simulation of wave travel does not consist of one time step, but a certain time $t$ is needed for the pressure pulse to propagate through the computational domain, given by the ratio of the length of the domain to the speed of the wave. As $t / \Delta t$ consecutive time steps are needed for the simulation of this propagation, only a factor $|G|^{t / \Delta t}$ of the original pulse will remain after the last time step. The numerical dissipation after a propagation time $t$ is thus given by

$$
1-|G|^{t / \Delta t}
$$

Note that if one is interested in capturing the reflections at the boundaries of the computational domain, also their propagation time should be considered in the calculation of the total numerical dissipation. 


\subsection{Numerical study on the hemodynamic impact of local stiffening}

The results from the previous studies are finally used to determine the settings necessary to obtain a grid and time step converged simulation of the wave propagation and reflection in a model of an aorta with repaired aortic coarctation. As the high level of complexity, inherent in patient-specific aortic models, may hamper the insights provided by these models, a simplified model of repaired aortic coarctation is used.

\subsubsection{FSI model}
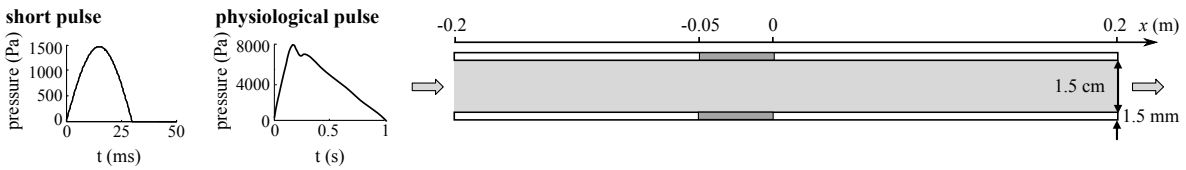

Figure 2: Simplified three-dimensional model of an aorta with repaired aortic coarctation.

The aorta is represented by a three-dimensional flexible tube and the effect of the repaired aortic coarctation is modeled by including a $5 \mathrm{~cm}$ long stiff segment, $15 \mathrm{~cm}$ distal to the inlet (see Fig. 2).

Two cases are studied. In the first case, the positive half of a short sinusoidal pressure pulse (period $T$ of $60 \mathrm{~ms}$, amplitude of $1470 \mathrm{~Pa}$ which is representative for the foot of a physiological pulse) is imposed at the inlet. A linear elastic material model is used to describe the wall behavior (Young's modulus $250 \mathrm{kPa}$, Poisson's ratio 0.49 , density $1200 \mathrm{~kg} / \mathrm{m}^{3}$ ). The stiff segment is chosen six times stiffer than the flexible wall (Young's modulus $1500 \mathrm{kPa}$ ). In the second case, a physiological pressure pulse (period $T$ of $1 \mathrm{~s}$, amplitude of approximately $60 \mathrm{mmHg}$ ) is used as an inlet boundary condition. As the deformations become more pronounced in this case, a hyperelastic material model is used (a reduced form of the generalized Mooney-Rivlin) with experimentally obtained coefficients for the human aorta $\left(\mathrm{C}_{10}=18.9, \mathrm{C}_{01}=2.75, \mathrm{C}_{20}=590.4, \mathrm{C}_{11}=\right.$ 857.18 and $\mathrm{C}_{30}=0 \mathrm{kPa}$ ) [24]. The behavior of the local stiffening is modeled using a linear elastic model (Young's modulus $1500 \mathrm{kPa}$ ).

In both cases a reflection free boundary is used ( at the outlet of the fluid domain to isolate the effects of reflections due to the presence of the stiff segment.

\subsubsection{Wave separation}

To analyze the wave reflections generated by the local stiffening, the pressure waves are decomposed into their forward and backward propagating components. A condensed version of the mathematics behind wave separation is presented. More details can be found in [25].

If $\Delta p_{ \pm}$and $\Delta v_{ \pm}$represent the changes in pressure and velocity in the forward ' + and backward '-' waves, additivity requires

$$
\Delta p=\Delta p_{+}+\Delta p_{-}
$$




$$
\Delta v=\Delta v_{+}+\Delta v_{-} .
$$

From the characteristic decomposition it follows that a simple relation exists between the changes in pressure and velocity in any wavefront, given by the water hammer equations.

$$
\Delta p_{ \pm}= \pm \rho_{f} c \Delta v_{ \pm}
$$

Eqs. (27a), (27b) and (28) can be solved for the forward and backward pressure changes. This results in

$$
\Delta p_{ \pm}=\frac{1}{2}\left(\Delta p \pm \rho_{f} c \Delta v\right) .
$$

The forward and backward pressure waves are finally calculated by summing these wave fronts

$$
p_{ \pm}(t)=\sum_{i=0}^{t} \Delta p_{ \pm}(i) .
$$

As the flow is initially at rest, no pressure waves are present at $t=0$.

\section{Results}

\subsection{Numerical study}

The importance of a proper time step size is illustrated in Figure 3. This figure shows the pressure contours along the tube obtained for a simulation with a low (top) and a high temporal resolution (bottom). A strong decrease in amplitude during wave propagation can be observed for the simulation with the lowest temporal resolution and inaccurate results are obtained due to numerical dissipation.

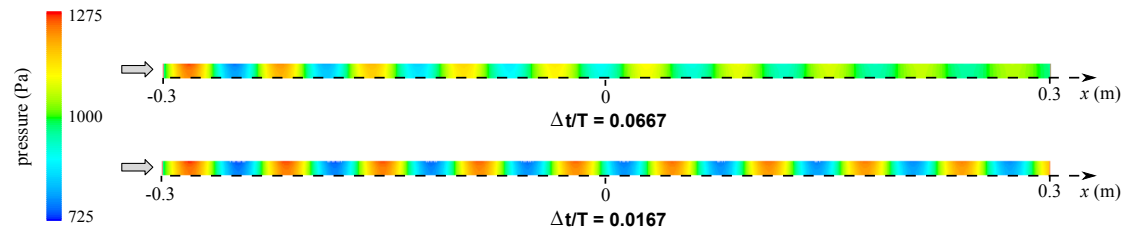

Figure 3: Simulation of the wave propagation in a two-dimensional axisymmetric tube obtained with (top) a low and (bottom) a high temporal resolution. Pressure contours $(\mathrm{Pa})$ in the fluid interior obtained using a second-order implicit time integration scheme.

The simulation of the wave propagation is performed for different values of $\Delta t / T$, ranging from 0.0667 to 0.0042 . Besides numerical dissipation of the pressure pulse, also viscous dissipation occurs during propagation. The numerical dissipation can thus not simply be determined by considering the reduction in amplitude of the pressure wave. As the amplitudes of the propagating waves, simulated with $\Delta t / T$ equal to 0.0083 and 0.0042 are almost equal, it can be concluded that the numerical dissipation becomes negligible using a value of 0.0042 for $\Delta t / T$. In that case the amplitude 
reduction is considered to be only due to viscous dissipation. As the viscous dissipation after a time $t$ is independent of the time step size, additional amplitude reductions in the simulations with a larger time step size are caused by numerical dissipation.

To calculate the numerical dissipation after a propagation time $t$, the relative reduction in amplitude $\phi$ of the time periodic wave at two positions $\left(x_{1}\right.$ and $\left.x_{2}\right)$ along the tube is considered. These positions are chosen such that the time for the wave to propagate with a speed $c$ from point $x_{1}$ to $x_{2}$ is equal to $t$. To clear out the effect of viscous dissipation, the relative amplitude reduction obtained for the time step size independent simulation is subtracted from this relative amplitude reduction. Using a value for $\Delta t / T$ equal to $\delta$, the numerical dissipation after a propagation time $t$, is thus calculated by

$$
\frac{\left[\phi\left(x_{2}\right) / \phi\left(x_{1}\right)\right]_{\Delta t / T=0.0042}-\left[\phi\left(x_{2}\right) / \phi\left(x_{1}\right)\right]_{\Delta t / T=\delta}}{\left[\phi\left(x_{2}\right) / \phi\left(x_{1}\right)\right]_{\Delta t / T=0.0042}} .
$$

Fig. 4 shows the thus obtained numerical dissipation as a function of the temporal resolution of the wave. The numerical dissipation increases more than linearly with increasing $\Delta t / T$.

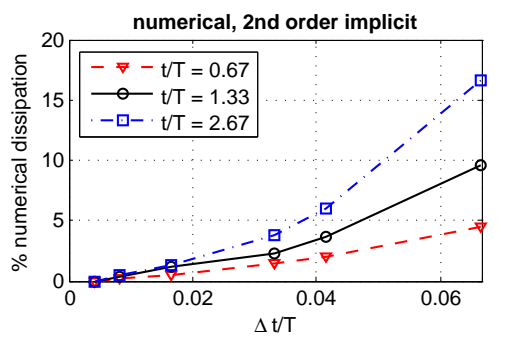

Figure 4: The numerical dissipation after a propagation time $t$ as a function of $\Delta t / T$ for different values of $t / T(\Delta x / \lambda=0.0067)$.

Similar, simulations with different values of $\Delta x / \lambda$ ranging from 0.0027 to 0.044 are performed. Grid independent results are obtained for a value of $\Delta x / \lambda$ equal to 0.0027 and this simulation is thus used as a reference for the other simulations. Using a value of $\Delta x / \lambda$ equal to $\delta$, the numerical dissipation after a propagation time $t$, is thus calculated by

$$
\frac{\left[\phi\left(x_{2}\right) / \phi\left(x_{1}\right)\right]_{\Delta x / \lambda=0.0027}-\left[\phi\left(x_{2}\right) / \phi\left(x_{1}\right)\right]_{\Delta x / \lambda=\delta}}{\left[\phi\left(x_{2}\right) / \phi\left(x_{1}\right)\right]_{\Delta x / \lambda=0.0027}} .
$$

In Fig. 5 the effect of $\Delta x / \lambda$ on the numerical dissipation after a propagation time $t$ is shown for different values of $t / T$. It can be seen that the numerical dissipation increases more than linearly with increasing $\Delta x / \lambda$. For the configuration studied here (secondorder accurate in space and time), a relatively low temporal and spatial resolution can be sufficient to restrict the numerical dissipation. Since in cardiovascular simulations the grid size is typically a few orders smaller than the wavelength of the present wave harmonics (approximately ranging from 5 to $0.25 \mathrm{~m}$ ), the spatial resolution will have no significant effect on the numerical dissipation. Only for very short pulses, the grid size may become influential. 


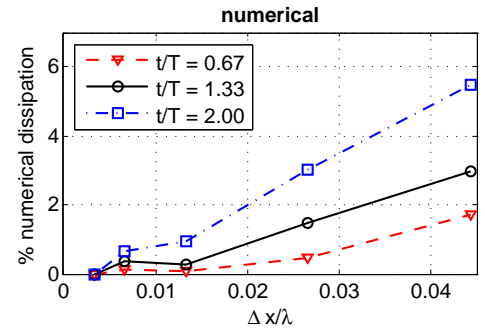

Figure 5: The numerical dissipation after a propagation time $t$ as a function of $\Delta x / \lambda$ for different values of $t / T(\Delta t / T=0.0167)$.

Figs. 4 and 5 demonstrate that the propagation time $t$ has a large impact on the total numerical dissipation.

\subsection{Analytical study}

Fig 6 depicts the relation between the temporal resolution of a wave and the numerical dissipation after a propagation time $t$ as derived in the analytical study and compares the results obtained with a first-order scheme (left) to the ones obtained with a second-order scheme (right). According to this figure the numerical dissipation increases less than linearly with decreasing temporal resolution for the first-order scheme whereas for the second-order scheme, a more than linear increase can be observed. On the other hand, a much higher temporal resolution is required to obtain the same degree of numerical dissipation. For example, to limit the numerical dissipation after a time $t / T=2.67$ to $5 \%$, a temporal resolution of 1000 time steps per period is needed for the simulation using the first-order scheme while only 27 time steps per period are required for the simulation using the second-order scheme. Compared to the results derived in the numerical study (Figure 4), the same nonlinear relationship between the numerical dissipation and $\Delta t / T$ can be observed and a qualitative and quantitative agreement is found.
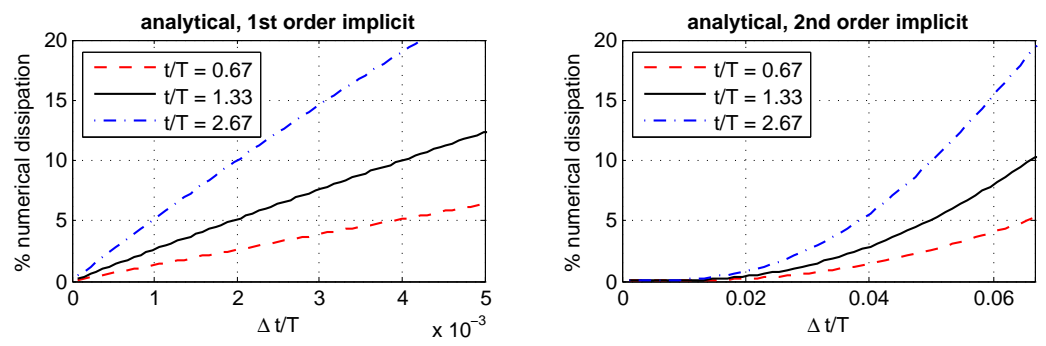

Figure 6: The grid independent numerical dissipation after a propagation time $t$ as a function of $\Delta t / T$ for different values of $t / T$ obtained using (left) a first-order and (right) a second-order implicit scheme. 
Fig. 7 depicts the effect of the spatial resolution on the numerical dissipation after a propagation time $t$ for different values of $t / T$. Compared to the numerical results (Fig. 5 ), the analytical results slightly overestimate the numerical dissipation. The analytical study, however, considers the damping in time of a wave that remains periodic in space, whereas in the numerical study, the dissipation is analyzed by studying the reduction in amplitude of a wave while it propagates along the tube and is thus not periodic in space. One should keep in mind, however, that the analytical study is only a guidance for the selection of the numerical parameters as is considers a very simplified model of the blood flow in an artery.

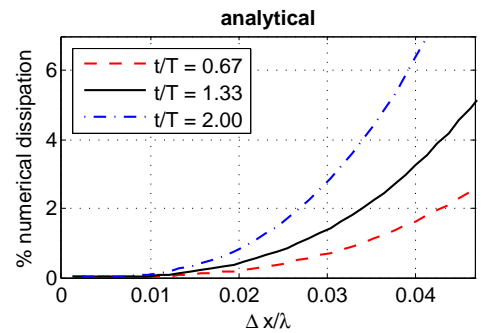

Figure 7: The time step size independent numerical dissipation after a propagation time $t$ as a function of $\Delta x / \lambda$ for different values of $t / T$.

Although the period $T$ and the speed of propagation $c$ of the wave harmonics present in the system can mostly not be chosen arbitrarily, attention should be paid if their values are altered (which is for example the case during exercise or when the stiffening of the arterial wall is modeled). Fig. 8 (right) shows the numerical dissipation after one time step as a function of the period of the wave and the wave speed for a fixed $\Delta x$ and $\Delta t$. Since both $\Delta t / T$ and $\Delta x / \lambda$ decrease with increasing wave period, the numerical dissipation will decrease exponentially with increasing period. A decrease of the wave speed leads to a reduced spatial resolution of the pressure wave (for a particular $T$ and $\Delta x$ ). This reduced spatial resolution results in a slightly increased numerical dissipation.

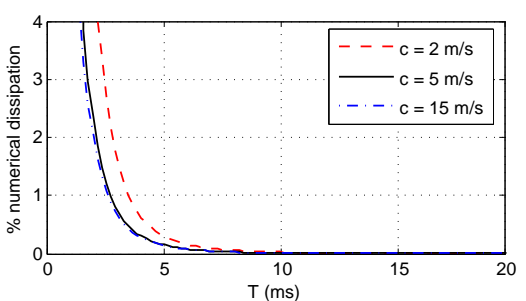

Figure 8: The numerical dissipation after one time step as a function of the period $T$ of a sinusoidal wave for different wave speeds $c(\Delta t=0.25 \mathrm{~ms}, \Delta x=0.5 \mathrm{~mm})$. 


\subsection{Numerical study on the hemodynamic impact of local stiffening}

\subsubsection{Selection of the numerical parameters}

Fig. 9 depicts the effect of the accuracy of the time integration scheme and illustrates the importance of a proper time step size selection when simulating the propagation of a short pulse in a locally stiffened artery (using the model from Figure 2). As these simulations involve the propagation of a pressure pulse, different wave harmonics will be present in the system. The lower the lower the frequency of the wave component, the better this wave is resolved in space and time and the more accurate it is simulated. Fig. 9 shows the pressure evolution along the artery at different times $t$ during the propagation. A coarse time step size of $4 \mathrm{~ms}$ is used to simulate the results on the left, whereas the results on the middle and the right are generated with a small time step size of 1 and $0.25 \mathrm{~ms}$ respectively. As demonstrated in the analytical study, the simulation using the first-order scheme requires a much higher temporal resolution to obtain the same degree of numerical dissipation. As such, smaller time steps (2, 0.25 and $0.1 \mathrm{~ms}$ ) are applied for this simulation. A strong decrease in amplitude during wave propagation can be observed for the simulations with the lowest temporal resolution. Another effect of the use of a coarse time step size is the damping of the wave reflections proximal to the stiff segment (indicated with an arrow in the figure on the right).
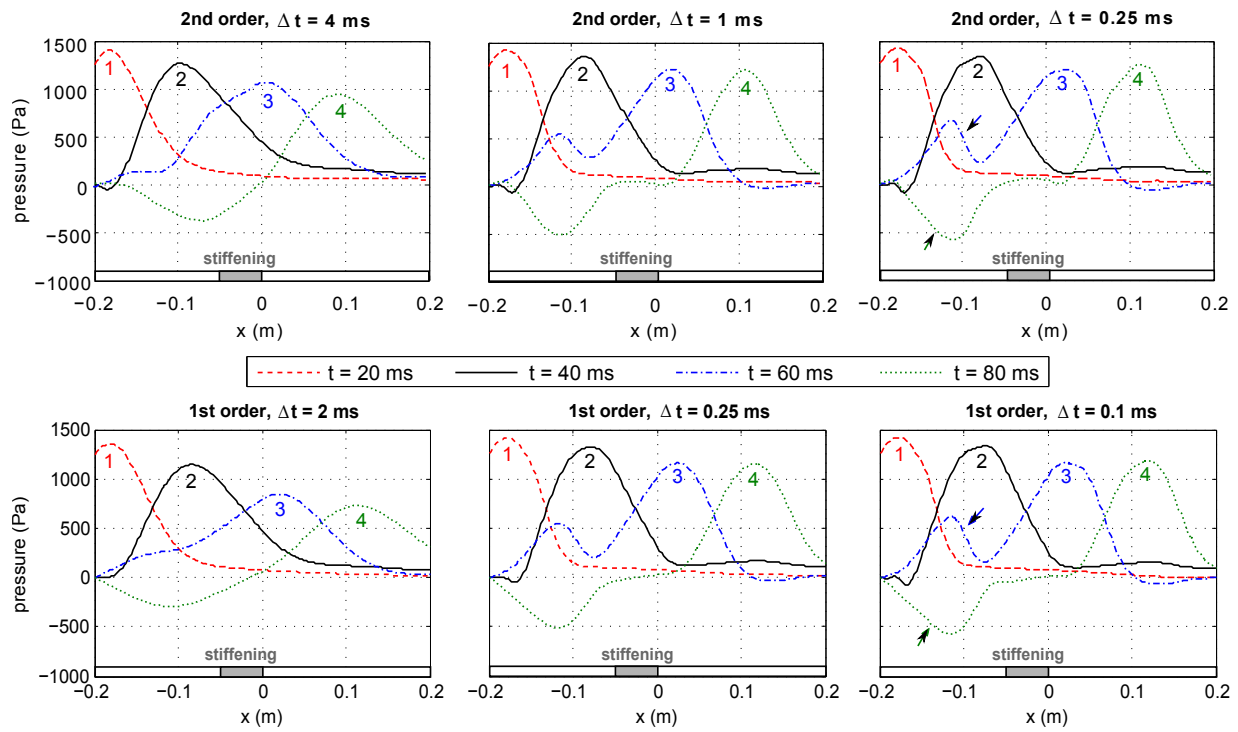

Figure 9: Simulation of the propagation of a short pressure pulse in a locally stiffened tube using different time integration schemes (second-order, top, versus first-order implicit, bottom) and different time step sizes.

Next, the hemodynamic impact of a local stiffening is studied for the propagation of a short and a physiological pressure pulse. In the first case, a time step size of 0.25 $\mathrm{ms}$ will be used. In the second case, a time step size of $5 \mathrm{~ms}$ is chosen, as the numerical 
dissipation is less significant due to the large pulse period (1s). The fluid domain is meshed using a structured grid with approximately 28000 cells. 1500 quadratic continuum elements are used to discretize the arterial wall.

\subsubsection{Wave separation analysis: short pressure pulse}

In the straight tube, the presence of the stiff segment induces two backward waves. At the transition from the elastic tube to the stiff segment, the wave speed suddenly increases, causing the forward wave to be amplified and a backward compression wave $(\mathrm{BCW})$ to be generated. The moment the pressure pulse leaves the stiff segment, the wave speed decreases again, causing the forward wave to be reduced and a backward expansion wave (BEW) to be created. This is demonstrated in Fig. 10 (top), which shows the pressure and its forward and backward component proximal to (left), halfway (middle) and distal to (right) the stiff segment.
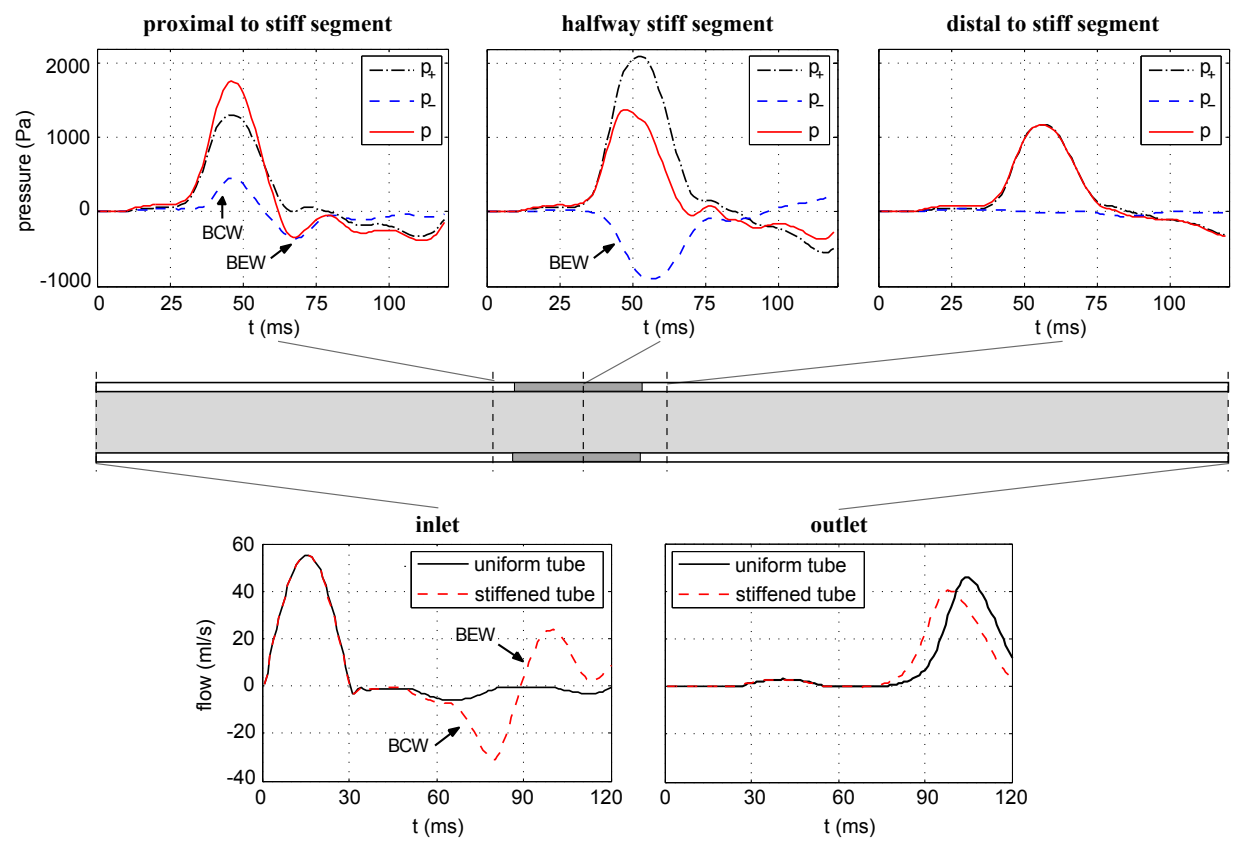

Figure 10: (top) Evolution of the pressure $p$ and its forward $p_{+}$and backward $p_{-}$component proximal to, halfway and distal to the stiffening for the case of a short pressure pulse. (bottom) Effect of a local stiffening on the inflow (left) and the outflow (right).

Proximal to the stiff segment these two backward waves overlap with a time delay $\tau$ given by

$$
\tau=\frac{2 L_{\text {stiffening }}}{c_{\text {stiffening }}}=8.4 \mathrm{~ms}
$$

where $L_{\text {stiffening }}$ represents the length of the stiff segment and $c_{\text {stiffening }}$ the local wave speed. 
The overall comparison with the flow in a uniform tube, representing a healthy aorta, is shown in Fig. 10 (bottom). The presence of the stiffening significantly alters the flow at the inlet of the model. The backward compression and expansion wave generated by the stiff segment can clearly be observed. At the outlet a small time delay between both pressure waves is visible, due to the increased wave speed in the stiff segment. The stiff segment reduces the amplitude of the flow wave by $11 \%$ compared to a straight tube.

\subsubsection{Wave separation analysis: physiological pressure pulse}

Fig. 11 (top) shows the pressure and its forward and backward component proximal to (left), halfway (middle) and distal to (right) the stiff segment for the propagation of a physiological pressure pulse. Proximal to the stiffening the backward compression and expansion wave overlap and cancel each other out. As a result, only a small peak in the backward component can be distinguished (BCW), caused by the time delay between both waves. The backward expansion wave (BEW), generated at the distal end of the stiff segment, is clearly visible in Fig. 11 (middle).

As can be seen in Fig. 11 (bottom), the local stiffening has almost no effect on the hemodynamic response in case a physiological pressure pulse is imposed at the inlet. Only for the flow at the inlet of the model small changes are found.
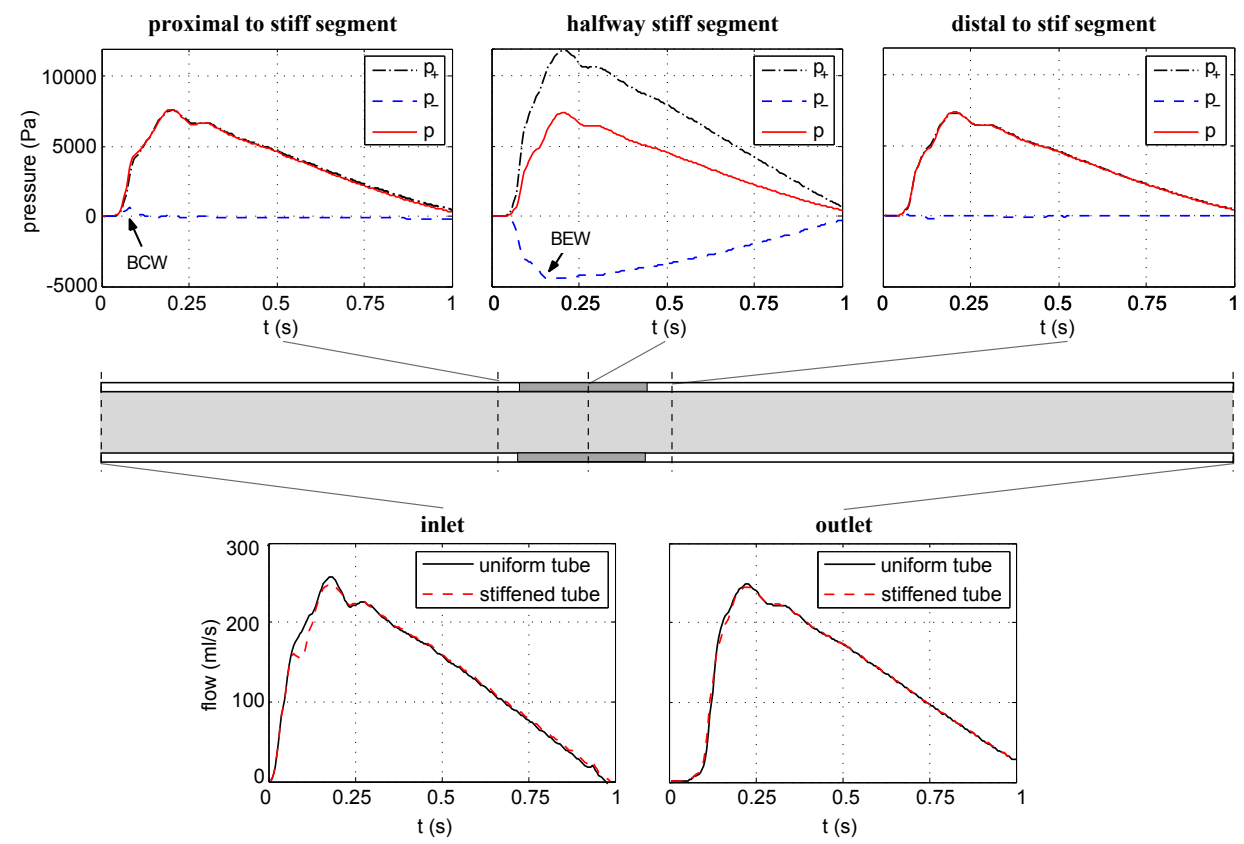

Figure 11: (top) Evolution of the pressure $p$ and its forward $p_{+}$and backward $p_{-}$component proximal to, halfway and distal to the stiff segment for the case of a physiological pressure pulse. (bottom) Effect of a local stiffening on the inflow (left) and the outflow (right). 


\section{Discussion and conclusion}

When solving a differential equation by a numerical approximation method, numerical dissipation and dispersion may occur. The study of these errors and their effect on the solution is a well-documented topic, but is sometimes overlooked in FSI simulations, where the numerical parameters are often optimized with respect to time efficiency and stability rather than accuracy $[15,16]$. Numerical dissipation is a general problem, occurring both in monolithic and partitioned FSI simulations. This papers aims to elucidate the effect of numerical dissipation on the solution of cardiovascular FSI simulations using both numerical simulations of the blood flow in an artery and an analytical study in which the scalar linear one-dimensional transport equation is considered.

The numerical dissipation depends on three parameters: $\Delta t / T$ and $\Delta x / \lambda$, determining the temporal and spatial resolution of the wave harmonics and $t / \Delta t$, defining the number of consecutive time steps during which the dissipation occurs. It is shown that for a second-order upwind discretization scheme in combination with a second-order implicit time integration scheme, the numerical dissipation after a propagation time $t$ increases more than linearly with increasing $\Delta t / T$ (Fig. 4 and Fig. 6 right) and with increasing $\Delta x / \lambda$ (Fig. 5 and Fig. 7). Furthermore, the comparison is made with a simulation for which a first-order implicit time integration scheme is used, demonstrating the need for a much higher temporal resolution in the latter case (Fig. 6 and Fig. 9). Since in cardiovascular simulations, the grid size is typically a few orders smaller than the wavelength of the present wave harmonics, the spatial resolution will have no significant effect on the numerical dissipation.

As both the time step size and the grid size can affect the numerical results considerably, their influence should be examined at the start of the simulation. This can be done analytically and/or numerically. The analytical study in which a simplified model of the problem is applied can be used as a guidance for the selection of the discretization parameters. The final choice of these parameters should preferable be made by studying the accuracy of the numerical simulations in which the full complexity of the problem is considered. When the pulse period and/or the wavelength change (which is the case during exercise) or the propagation time increases the grid size and/or the time step size need to be adapted in order to obtain the same degree of numerical dissipation.

In addition, the grid and time step converged wave propagation in an aorta with repaired coarctation is studied. While other FSI studies on aortic coarctation focus on the reduction of the cardiac load when comparing the pre-intervention and postintervention hemodynamic conditions [26] or the hemodynamic impact of surgical reconstructed aortic arches [27], this analysis focuses on the reflections generated by the local stiffening remaining after the treatment of aortic coarctation.

While patient-specific FSI models have the convenience of taking into account the full complexity of the problem under consideration, their high level of complexity may also hamper the analysis and insights provided. In this work, a very simplified model of repaired aortic coarctation was used. The advantage of this model is that the only reflections present in the model originate from the local stiffening and can thus easily be interpreted. However, to obtain a complete analysis, a patient-specific geometry should be applied, including the residual narrowing, which often remains after the treatment 
of aortic coarctation. Besides discrete reflections, generated at the branching of the aorta and by the residual narrowing and stiffening, also scattered wave reflections will be present, caused by the tapering of the aorta.

In a first case the propagation of a short pressure pulse is simulated. The main motivation for the study of this setup, is that the different reflections become clearly distinguishable. Indeed, the time delay $\tau$ between both waves is of the same magnitude as the duration of the pulse itself ( 8.4 versus $30 \mathrm{~ms}$ ). The simulation of a short pulse consequently improves the understanding of the phenomena involved.

The simulation of a physiological pressure pulse, on the other hand, can be used to predict the actual hemodynamic impact of a local stiffening. Although the simulation of the short pulse suggests quite an important alteration of the hemodynamic quantities, no significant changes are found for the case of a physiological pressure pulse. This counter-intuitive result can, nonetheless, be explained by recalling the reflections induced by the stiff segment. At the transition from the flexible artery to the stiffening, a backward compression wave is generated, immediately followed by a backward expansion wave, created at the outlet of the stiffening. Proximal to the stiff segment, both waves overlap and cancel each other out, as such leaving the hemodynamic quantities almost undisturbed.

These results are in agreement with the results of the one-dimensional studies published in $[28,29]$. In these studies a 1D model was developed to predict the hemodynamic impact of a stent, modeled as a local stiffening. Both studies concluded that the major effect of the presence of the stent is a small pressure build-up proximal to the stent. Increased stiffness and length of the prosthesis amplified this effect. In [28], the magnitude of the reflected wave was less than $1 \%$ of the normal pulse pressure, for all cases tested. In [29] larger pressure raises (up to 12\%) were reported. However, this study did not use physiologically correct values for the dimensions and physical properties.

In practice, more than two backward waves are created. Particularly, the BEW generated at the distal end of the stiffening, will reflect in a forward compression wave $(\mathrm{FCW})$ at the proximal end of the stiffening. This wave will re-reflect at the distal end of the stiff segment, creating a second BEW, which in turn, generates a second FCW and so on. As such, part of the wave energy will reach the outlet with a delay (as a sequence of compression waves). This explains the amplitude reduction at the outlet, observed in Figure 10. These phenomena are clearly illustrated in [30], in which the propagation of a $2.5 \mathrm{~ms}$ wide pulse is studied. Considering that each reflection is just a fraction of the original wave and the time delay between the different reflections is very small compared to the pulse period, these secondary reflections are not individually observable in the studied examples.

\section{References}

[1] J. Murgo, N. Westerhof, J. Giolma, S. Altobelli, Aortic input impedance in normal man: relationship to pressure wave forms, Circulation 62 (1980) 105-116.

[2] M. O'Rourke, Mechanical principles. Arterial stiffness and wave reflection, Pathol. Biol. (Paris) 47 (1999) 623-633. 
[3] M. O'Rourke, W. Nichols, Changes in wave reflection with advancing age in normal subjects, Hypertension 44 (2004) E10-E11.

[4] G. Agnoletti, C. Bonnet, D. Bonnet, D. Sidi, Y. Aggoun, Mid-term effects of implanting stents for relief of aortic recoarctation on systemic hypertension, carotid mechanical properties, intimal medial thickness and reflection of the pulse wave, Cardiol. Young 15 (3) (2005) 245-250.

[5] R. Beekman, D. Muller, P. Reynolds, C. Moorehead, K. Heideberger, F. Lupinetti, Balloon-expandable stent treatment of experimental coarctation of the aorta early hemodynamic and pathological evaluation, J. Interv. Cardiol. 6 (1993) 113123.

[6] G. Wright, C. Nowak, C. Goldberg, R. Ohye, E. Bove, A. Rocchini, Extended resection and end-to-end anastomosis for aortic coarctation in infants: Results of a tailored surgical approach, Ann. Thorac. Surg. 80 (4) (2005) 1453-1459.

[7] T. Murakami, A. Takeda, Enhanced aortic pressure wave reflection in patients after repair of aortic coarctation, Ann. Thorac. Surg. 80 (3) (2005) 995-1000.

[8] S. Sherwin, V. Franke, J. Peiro, K. Parker, One-dimensional modelling of a vascular network in space-time variables, J. Eng. Math. 47 (2003) 217-250.

[9] K. Azer, C. Peskin, A one-dimensional model of blood flow in arteries with friction and convection based on the womersley velocity profile, Cardiovasc. Eng. 7 (2007) 51-73.

[10] A. Avolio, Multi-branched model of the human arterial system, Med. Biol. Eng. Comput. 18 (1980) 709-718.

[11] F. van de Vosse, N. Stergiopulos, Pulse wave propagation in the arterial tree, Annu. Rev. Fluid Mech. 43 (2010) 467-499.

[12] C. Taylor, C. Figueroa, Patient-specific modeling of cardiovascular mechanics, Annu. Rev. Biomed. Eng. 11 (2009) 109-34.

[13] F. van de Vosse, J. de Hart, C. van Oijen, D. Bessems, T. Gunther, Finite-elementbased computational methods for cardiovascular fluid-structure interaction, J. Eng. Math. 47 (2003) 335-68.

[14] P. Crosetto, P. Reymond, S. Deparis, D. Kontaxakis, N. Stergiopulos, A. Quarteroni, Fluid-structure interaction simulation of aortic blood flow, Comput. Fluids 43 (2011) 46-57.

[15] J. Janela, A. Moura, A. Sequeira, Absorbing boundary conditions for a 3D nonNewtonian fluid-structure interaction model for blood flow in arteries, Int. J. Eng. Sc. 48 (2010) 1332-1349.

[16] E. Jarvinen, M. Lyly, J. Ruokolainen, P. Raback, Three-dimensional fluidstructure interaction modeling of blood flow in elastic arteries, in: ECCOMAS CFD, Swansea, United Kingdom, 2001. 
[17] E. Isaacson, H. Keller, Analysis of Numerical Methods, Dover, 1994.

[18] A. Quarteroni, Numerical Models for Differential Problems, Springer, 2009.

[19] J. Ferziger, M. Peric, Computational Methods for Fluid Dynamics, Springer, 2002.

[20] S. V. Patankar, Numerical Heat Transfer and Fluid Flow, Hemisphere, 1980.

[21] H. M. Hilber, T. J. R. Hughes, R. L. Taylor, Improved numerical dissipation for time integration algorithms in structural dynamics, Earthq. Eng. Struct. Dyn. 5 (1977) 283-292.

[22] J. Degroote, K.-J. Bathe, J. Vierendeels, Performance of a new partitioned procedure versus a monolithic procedure in fluid-structure interaction, Comput. Struct. 87 (11-12) (2009) 793-801.

[23] R. LeVeque, Numerical Methods for Conservation Laws, Birkhäuser, 1992.

[24] P. Prendergast, C. Lally, S. Daly, A. Reid, T. Lee, D. Quinn, F. Dolan, Analysis of prolapse in cardiovascular stents: A constitutive equation for vascular tissue and finite-element modelling, J. Biomech. Eng. - T. ASME 125 (2003) 692-699.

[25] K. Parker, An introduction to wave intensity analysis, Med. Biol. Eng. Comput. 47 (2009) 175-188.

[26] H. J. Kim, I. E. Vignon-Clementel, C. A. Figueroa, J. F. LaDisa, K. E. Jansen, J. A. Feinstein, C. A. Taylor, On coupling a lumped parameter heart model and a three-dimensional finite element aorta model, Ann. Biomed. Eng. 37 (11) (2009) 2153-2169.

[27] S. Pittaccio, F. Migliavacca, R. Balossino, G. Dubini, E.-T. Frund, V. Hjortdal, M. Smerup, E. Morre-Pedersen, M. R. De Leval, MRI-based multiscale models for the hemodynamic and structural evaluation of surgically reconstructed aortic arches, J. Appl. Biomat. Biom. 5 (1) (2007) 11-22.

[28] J. Charonko, S. Ragab, P. Vlachos, A scaling parameter for predicting pressure wave reflection in stented arteries, Journal of Medical Devices - T. ASME 3 (2009) 10.

[29] L. Formaggia, F. Nobile, A. Quarteroni, A one dimensional model for blood flow: Application to vascular prosthesis, in: Mathematical Modeling and Numerical Simulation in Continuum Mechanics, Springer-Verlag Berlin, Berlin, 2002, pp. 137-153.

[30] L. Formaggia, A. Veneziani, Reduced and multiscale models for the human cardiovascular system, Lecture notes VKI Lecture Series 2003-07, Brussels, 2003, Ch. 1.3.3. 